\title{
Large-scale numerical modeling of hydro-acoustic waves generated by tsunamigenic earthquakes
}

\author{
C. Cecioni ${ }^{1}$, A. Abdolali ${ }^{1,2}$, G. Bellotti ${ }^{1}$, and P. Sammarco ${ }^{3}$ \\ ${ }^{1}$ Roma Tre University, Engineering Department, Via Vito Volterra 62, 00146 Rome, Italy \\ ${ }^{2}$ Department of Civil and Environmental Engineering, Center for Applied Coastal Research, \\ University of Delaware, Newark, Delaware, USA \\ ${ }^{3}$ University of Rome Tor Vergata, Civil Engineering Department, Via del Politecnico 1, 00133 Rome, Italy \\ Correspondence to: C. Cecioni (claudia.cecioni@uniroma3.it)
}

Received: 26 May 2014 - Published in Nat. Hazards Earth Syst. Sci. Discuss.: 11 July 2014

Revised: - - Accepted: 9 March 2015 - Published: 24 March 2015

\begin{abstract}
Tsunamigenic fast movements of the seabed generate pressure waves in weakly compressible seawater, namely hydro-acoustic waves, which travel at the sound celerity in water (about $1500 \mathrm{~m} \mathrm{~s}^{-1}$ ). These waves travel much faster than the counterpart long free-surface gravity waves and contain significant information on the source. Measurement of hydro-acoustic waves can therefore anticipate the tsunami arrival and significantly improve the capability of tsunami early warning systems. In this paper a novel numerical model for reproduction of hydro-acoustic waves is applied to analyze the generation and propagation in real bathymetry of these pressure perturbations for two historical catastrophic earthquake scenarios in Mediterranean Sea. The model is based on the solution of a depth-integrated equation, and therefore results are computationally efficient in reconstructing the hydro-acoustic waves propagation scenarios.
\end{abstract}

\section{Introduction}

Submarine earthquakes are the major cause of generation of tsunami. A correct modeling of the wave field generated by seabed movement is mandatory to understand the physics of the tsunami and its propagation. Most of the hydraulic tsunami models make use of the water incompressibility hypothesis. Nevertheless a sudden movement of the seabed, triggered by underwater earthquake, compresses the water column and generates pressure waves (hydroacoustic waves) that propagate in the sea at the celerity of sound in water. The present analysis involves only the first- order effects of compressibility on the generation of surface (tsunami) and hydro-acoustic waves, and does not consider the secondary wave-to-wave interaction (e.g., pseudoRayleigh and acoustic-gravity waves considered in the work of Ardhuin and Herbers, 2013).

Since the hydro-acoustic waves travel much faster than the surface waves, their real-time recording allows the anticipation of the tsunami arrival. Moreover, the hydro-acoustic wave signals contain significant information on the tsunamigenic source (Cecioni et al., 2014; Chierici et al., 2010). Therefore the detection and the complete modeling of hydroacoustic waves (speed around $1500 \mathrm{~m} \mathrm{~s}^{-1}$ ), together with measurements of seismic waves (speed around $5000 \mathrm{~m} \mathrm{~s}^{-1}$ ), could in principle dramatically improve the effectiveness of tsunami early warning systems.

Earlier studies on tsunami evolution in weakly compressible water have been carried out by Miyoshi (1954), Sells (1965) and Yamamoto (1982). Later, analytical studies have solved the potential fluid problem in weakly compressible water, with fast-rising motion of the seabed (Nosov, 1999; Stiassnie, 2010; Chierici et al., 2010). The analytical models, although extremely useful to investigate the features of hydro-acoustic wave generation and propagation processes, are not suited for reproduction of such phenomena at realgeographical scale. The numerical solution of the 3-D problem (Nosov and Kolesov, 2007; Bolshakova et al., 2011) is straightforward to obtain but requires unacceptable computational time when applied to large areas. A depth-integrated equation has therefore been proposed by Sammarco et al. (2013), which reproduces the mechanics of propagation of 
low-frequency hydro-acoustic waves due to a sudden bottom displacement associated with earthquakes. The wave simulation by means of this equation overcomes the computational difficulties of three-dimensional models and retains all the physical features of the phenomena. The model has been applied by Abdolali et al. (2015a) to reproduce the wave field generated by the 2012 Haida Gwaii tsunamigenic earthquake, and model results are compared with in situ wave measurements.

In this paper the results and the physical implications of a large geographical scale application of the numerical model based on the depth-integrated equation of Sammarco et al. (2013) are illustrated. The model is applied to simulate the hydro-acoustic wave propagation generated by two main tsunamigenic destructive historical earthquakes that occurred in the Mediterranean Sea: the AD 365 Crete event and the 1693 Sicily event. The depth-integrated model has been validated through comparison with the solution of the full three-dimensional weakly compressible wave problem in real bathymetry, along vertical sections of the sea. The comparison allowed some computational parameters to be set up in order to optimize the depth-integrated model.

The simulation of hydro-acoustic wave propagation in real bathymetry enables the investigation of the correlation between the hydro-acoustic waves and the generation mechanism, the source location, the bottom topography and the depth of the pressure recording point. The model is based on the hypothesis of rigid seabed; however, it is worth citing Eyov et al. (2013), who study the effects of replacing the rigid-bottom assumption by an elastic half-space solid medium on the properties of the acoustic-gravity waves. Chierici et al. (2010) investigated on the effect of a porous seabed, and more recently Abdolali et al. (2014) and Abdolali et al. (2015b) presented a depth-integrated equation to simulate gravity and hydro-acoustic waves over a viscous compressible sedimentary layer underlying seawater.

In the portion of the Mediterranean Sea considered in the present research, two deep-sea observatories are located off-shore of the eastern Sicilian coast. These observatories, described later in more details, are equipped, among others instruments, with low-frequency and large-bandwidth hydrophones. Therefore the numerically reproduced scenarios provide indications on the attended hydro-acoustic signals in the case of submarine earthquake occurrence.

The paper is structured as follows: the next section deals with the description of the numerical model; Sect. 3 describes the large-scale numerical simulations of the two selected historical tsunamis; in Sect. 4 discussions and conclusions are given.

\section{Description of the numerical model}

Consider the problem of wave propagation in weakly compressible inviscid fluid, where waves are generated by a seabed motion. In the framework of linearized theory the governing equation and boundary conditions for the fluid potential $\Phi(x, y, z, t)$ are

$$
\left\{\begin{array}{ll}
\Phi_{t t}-c_{\mathrm{s}}^{2} \nabla^{2} \Phi-c_{\mathrm{s}}^{2} \Phi_{z z}=0 & \\
\Phi_{t t}+g \Phi_{z}=0 & \text { at } \quad z=0 \\
\Phi_{z}+\nabla h \cdot \nabla \Phi+h_{t}=0 & \text { at } \quad z=-h(x, y, t)
\end{array},\right.
$$

where $\nabla$ and $\nabla^{2}$ are respectively the gradient and the Laplacian in the horizontal plane $x, y$, while subscript with the independent variables denotes partial derivatives, $c_{\mathrm{s}}$ is the celerity of sound in water, and $g$ is the gravity acceleration. $h$ is the water depth, given by the rest bottom topography $h_{\mathrm{b}}(x, y)$ net of the earthquake bottom motion $\zeta(x, y, t)$ :

$h(x, y, t)=h_{\mathrm{b}}(x, y)-\zeta(x, y, t)$.

From Eq. (2) $h_{\mathrm{t}}$, the water depth time variation, is zero everywhere except on the earthquake zone,

$h_{t}=-\zeta_{t}=-\frac{\zeta_{0}}{\tau}[H(t)-H(t-\tau)]$,

where $H$ is the Heaviside step function, $\zeta_{0}(x, y)$ is the residual displacement, $\tau$ is the duration of the seabed motion and the seabed velocity is assumed constant, as $\zeta_{0} / \tau$.

The solution of Eq. (1) is given by an infinite sum of natural modes, $n=0,1, \ldots$ (Eyov et al., 2013). The first mode $(n=0)$ represents the gravity surface wave, i.e., the tsunami; the other modes $(n \geq 1)$, namely the hydro-acoustic modes, are responsible for elastic oscillations of the water body. The hydro-acoustic waves are characterized by a set of natural frequencies, namely cutoff frequencies:

$f^{(n)}=\frac{(2 n-1) c_{\mathrm{s}}}{4 h}$,

where $n \geq 1$ is the indicator of the hydro-acoustic mode. Evanescent and progressive waves are associated respectively with lower and higher frequencies than the cutoff ones (Eq. 4).

Sammarco et al. (2013) via a proper application of the averaging technique to the problem (Eq. 1), in the hypothesis of constant $c_{\mathrm{s}}$ and mild sloped seabed $(\nabla h \ll k h)$, found a depth-integrated equation, which in hyperbolic form reads

$\psi_{n_{t t}}\left(\frac{C_{n}}{c_{\mathrm{s}}^{2}}+\frac{1}{g}\right)-\nabla\left(C_{n} \nabla \psi_{n}\right)+\left(\frac{\omega^{2}}{g}-\beta_{n}^{2} C_{n}\right) \psi_{n}=h_{t} D_{n}$,

where $\psi(x, y, t)$ is the fluid velocity potential at the undisturbed free surface. Equation (5) has been named a mildslope equation in weakly compressible fluid (MSEWC). The subscript $n$ indicates that Eq. (5) is valid for the generic $n$th mode (hydro-acoustic and gravity). Superimposition of the solutions of Eq. (5) for each mode will lead to complete modeling of the fluid potential, $\Phi(x, y, z, t)=$ $\sum_{n=0}^{\infty} \psi_{n}(x, y, t) f_{n}(z)$, generated by a fast seabed motion, 
Table 1. Magnitude $M_{\mathrm{w}}$ and seismic moment $M_{0}$ for the two scenarios as determined by Tonini et al. (2011).

\begin{tabular}{lll}
\hline Scenario & $M_{\mathrm{w}}$ & $M_{0}(\mathrm{Nm})$ \\
\hline AD 365 & 8.5 & $6.3210^{21}$ \\
1693 & 7.2 & $7.0710^{19}$ \\
\hline
\end{tabular}

where the $f_{n}$ 's are the classic eigenfunctions of the constant depth homogeneous problem, which is valid for mild-sloped seabed

$f_{n}(z)=\frac{\cos h\left[\beta_{n}(h+z)\right]}{\cosh \left(\beta_{n} h\right)}$.

In Eq. (5) $\beta_{n}$ 's are the roots of the dispersion relation

$\beta_{n}=\left\{\begin{array}{lll}n=0 & \beta_{n}=\beta_{0} & \omega^{2}=g \beta_{0} \tan h\left(\beta_{0} h\right) \\ n \geq 1 & \beta_{n}=i \overline{\beta_{n}} & \omega^{2}=-g \overline{\beta_{n}} \tan \left(\overline{\beta_{n}} h\right)\end{array}\right.$,

while the terms $C_{n}(x, y)$ and $D_{n}(x, y)$ are given by

$$
\begin{aligned}
C_{n}(x, y) & =\int_{-h}^{0} f_{n}^{2} \mathrm{~d} z=\frac{2 \beta_{n} h+\sin h\left(2 \beta_{n} h\right)}{4 \beta_{n} \cos h^{2}\left(\beta_{n} h\right)} \\
D_{n}(x, y) & =\frac{1}{\cosh ^{2}\left(\beta_{n} h\right)} \frac{\int_{-h}^{0} f_{n} \mathrm{~d} z}{\int_{-h}^{0} f_{n}^{2} \mathrm{~d} z} \\
& =\frac{4 \tan h\left(\beta_{n} h\right)}{\left(2 \beta_{n} h+\sin h\left(2 \beta_{n} h\right)\right)} .
\end{aligned}
$$

More details on the wave derivation can be found in Sammarco et al. (2013). Note that for incompressible fluid, i.e., in the limit $c_{\mathrm{s}} \longrightarrow \infty$, Eq. (5) reduces to the classical MSE (Berkhoff, 1972) extended to allow for bottom motion (Cecioni and Bellotti, 2010b, a). In the time domain the MSEWC reproduces the fluid potential characteristic of a narrowfrequency-banded wave spectrum, around the value of a carrier frequency, which determines the variables $\beta_{n}, C_{n}$ and $D_{n}$. For each narrow frequency band of the wave spectrum, Eq. (5) is solved and then the results are superimposed.

The numerical model makes use of the finite element method and solves the hyperbolic equation by means of a time-marching numerical scheme. The model uses the generalized- $\alpha$ method, which is a one-step implicit method for solving the transient problem. The method, first developed for the second-order equations in structural mechanics (Chung and Hulbert, 1993; Jansen et al., 2000), increases the amount of numerical damping present without degrading the order of accuracy.

\section{Mediterranean tsunami scenarios}

Two historical tsunami scenarios have been reproduced by means of the numerical solution of the depth-integrated

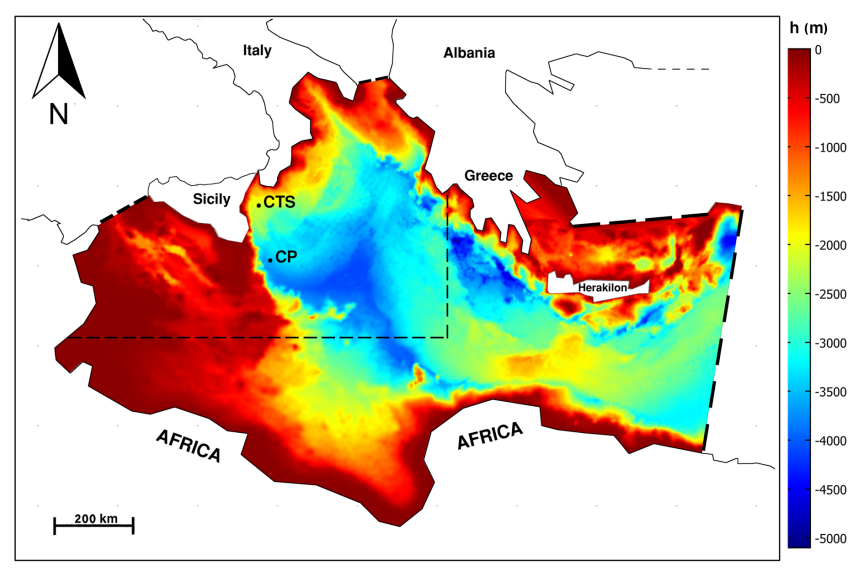

Figure 1. Mediterranean Sea bathymetry relative to the numerical domain.

MSEWC (Eq. 5). The selected tsunami events are those generated by the AD 365 Crete earthquake and the 1693 Sicily earthquake. We refer to the paper of Tonini et al. (2011), which estimates the tsunami hazard for the city of Catania (Sicily, Italy) by means of the Worst-case Credible Tsunami Scenario Analysis (WCTSA) technique. The authors have considered several possible earthquake scenarios of the two events of AD 365 and 1693 and performed a sensitivity analysis on the source size, since the choice of the source strongly affects the final results in terms of inundation map. The historical tsunami analyzed in the present study have been selected among the ones proposed by Tonini et al. (2011), as those generated by the largest reconstructed submerged earthquakes. Their seismic parameters are reported in Table 1. Figure 1 shows the domain of application of the numerical model, which is a portion of the Mediterranean Sea, and the relative bathymetry. The boundaries are the coasts of Italy, Greece and North Africa. Dashed black lines represent the open sea boundaries, where a condition of waves' free exit is imposed. For the 1693 Sicily tsunami scenario the numerical domain is restricted to the portion of Mediterranean Sea bounded by the thin dashed line. The data of the Mediterranean Sea bathymetry, represented in Fig. 1, are taken from ETOPO1, a 1 min bathymetric and relief database of the National Geophysical Data Center (NGDC). In Fig. 1 two points are indicated, namely CTS and CP, which correspond to the position of two submarine observatories, equipped with hydrophones. The Catania Test Site (CTS) observatory $\left(37^{\circ} 30.008^{\prime} \mathrm{N}, 15^{\circ} 23.034^{\prime} \mathrm{E}\right.$ northern site, $37^{\circ} 32.861^{\prime} \mathrm{N}, 15^{\circ} 23.844^{\prime}$ E southern site) is located around $25 \mathrm{~km}$ offshore of the harbor of Catania and at a water depth of $2 \mathrm{~km}$. The Capo Passero (CP) observatory $\left(36^{\circ} 17^{\prime} 33.57^{\prime \prime} \mathrm{N}, 15^{\circ} 58^{\prime} 53.02^{\prime \prime} \mathrm{E}\right)$, is located $100 \mathrm{~km}$ offshore of the Capo Passero village and at $3500 \mathrm{~m}$ below the mean sea level. Both observatories are connected to shore through a submarine electro-optical cable. The observatories located in the Catania Test Site are dedicated to multi- 
Table 2. Seismic parameters of AD 365 Crete earthquake, as reconstructed by Tonini et al. (2011), for the north (segment 1) and south (segment 2).

\begin{tabular}{lrr}
\hline Seismic parameters & Segment 1 & Segment 2 \\
\hline Length $(\mathrm{m})$ & 230 & 230 \\
Width $(\mathrm{m})$ & 90 & 90 \\
Strike $\left(^{\circ}\right)$ & 312 & 320 \\
Dip $\left(^{\circ}\right)$ & 21 & 21 \\
Rake $\left(^{\circ}\right)$ & 90 & 90 \\
Slip $(\mathrm{m})$ & 5 & 5 \\
Upper edge depth $(\mathrm{km})$ & 5 & 5 \\
\hline
\end{tabular}

disciplinary activities, equipped with low-frequency $(0.1 \mathrm{~Hz}-$ $1 \mathrm{kHz})$ and large-bandwidth $(10 \mathrm{~Hz}-70 \mathrm{kHz})$ hydrophones, seismometers, CTDs, magnetometers and current meters. The Capo Passero submarine observatory has been designed and constructed by INFN in the framework of the NEMO and KM3neT-Italia (www.km3net.org) projects. Aboard this observatory an antenna of large bandwidth hydrophone $(10 \mathrm{~Hz}-$ $70 \mathrm{kHz}$ ) has been installed within the activities of the SMO (Submarine Multidisciplinary Observatory) FIRB research project, funded by the Italian Ministry for University and Scientific Research (MIUR). SMO aims at collecting real-time acoustic data for three main scientific areas: astrophysics, for the neutrino detection, bio-acoustics, for the whale tracking, and geophysics for the recording of hydro-acoustic wave generated by submerged earthquakes. The SMO was installed in March 2013 and since then has been acquiring data in real time. The position of the two observatories has been used to analyze the numerically reproduced hydro-acoustic signals generated by the two historical earthquakes. Details on each earthquake-tsunami event and their modeling are given in the following subsections.

\subsection{The AD 365 scenario}

The AD 365 earthquake was an undersea earthquake that occurred on 21 July AD 365 in the eastern Mediterranean, with an assumed hypo-center located off western Crete, along a major thrust fault parallel to the western Hellenic Trench. Geologists today estimate that the quake intensity was 8.5 on the Richter scale or higher, causing widespread destruction in central and southern Greece, Libya, Egypt, Cyprus, and Sicily. In Crete nearly all towns were destroyed. This earthquake was followed by a tsunami which devastated the Mediterranean coasts, killing thousands of people and hurling ships $3 \mathrm{~km}$ inland.

This work considers a reconstructed scenario of this earthquake, in order to numerically reproduce the generated hydro-acoustic waves. Following the works of Shaw et al. (2008) and Tonini et al. (2011), we consider almost the whole rupture of the western Hellenic arc. Making use of the simplified fault model of Tonini et al. (2011), the seismic pa-

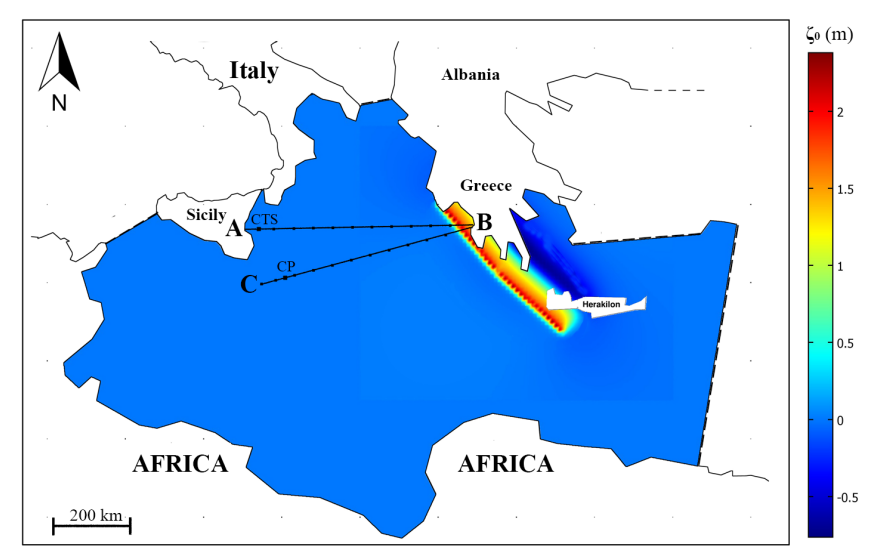

Figure 2. Residual vertical seabed dislocation, $\zeta_{0}(\mathrm{~m})$, for the AD 365 Crete earthquake as reconstructed by Tonini et al. (2011).

rameters are described in Table 2. The reconstructed residual seabed dislocation, $\zeta_{0}$, has been calculated by means of the Okada (1985) formulas and it is shown in Fig. 2.

In Fig. 2 two straight lines $A B$ and $C B$ are drawn: they pass through the two locations of the submarine observatories of Catania (CTS) and Capo Passero (CP). Along these two lines the MSEWC (Eq. 5) has been solved and compared with the solution of the complete 3-D problem (Eq. 1) applied to the two vertical sections ( $A B$ and $C B$ ). Figure 3 shows the water depth (upper panels) and the seabed residual vertical dislocations (lower panels) along these two cross sections. The comparison at the two cross sections reveals very good agreement between the depth-integrated and the 3-D models. For the sake of brevity just the results at the two points, CTS and $\mathrm{CP}$, are presented in Fig. 4. The panels a and b represent respectively the free-surface elevation time series and their frequency spectra at the CTS point, while the panels $\mathrm{c}$ and $\mathrm{d}$ are relative to the $\mathrm{CP}$ point. In each panel the upper plots show the solution of the 3-D reference model, and the lower plots show the depth-integrated model results. The time series are in very good agreement, although, as can be noted from their spectra (right column), not all the frequencies have been reproduced in the solution of the depth-integrated equation. In particular for the simulation here presented a set of MSEWC has been solved, each one relative to a frequency in the range $f=0.1-0.5 \mathrm{~Hz}$, with a $\Delta f=0.04 \mathrm{~Hz}$.

The comparison results have been used to set up some computational parameters of the large-scale simulation and to optimize the number of frequencies and number of modes to solve in order to obtain a good reproduction of the hydroacoustic signal, minimizing the computational costs. For the AD 365 earthquake scenario in the whole domain, the frequency range $f=0.1-0.5 \mathrm{~Hz}$, with a $\Delta f=0.04 \mathrm{~Hz}$, has been evaluated as sufficient in order to accurately reproduce the hydro-acoustic waves. It has been found that the first acoustic mode alone is responsible for carrying most of the energy transferred from the seabed motion. The large geo- 
Table 3. Seismic parameters of the 1693 Sicily earthquake, as reconstructed by Tonini et al. (2011), for the four segments from north to south.

\begin{tabular}{lrrrr}
\hline Seismic parameters & Segment 1 & Segment 2 & Segment 3 & Segment 4 \\
\hline Length (m) & 14.7 & 8.8 & 5 & 19.2 \\
Width (m) & 16.5 & 16.5 & 16.5 & 16.5 \\
Strike $\left(^{\circ}\right)$ & 329 & 322 & 343 & 360 \\
Dip $\left(^{\circ}\right)$ & 35 & 35 & 35 & 35 \\
Rake $\left({ }^{\circ}\right)$ & 270 & 270 & 270 & 270 \\
Slip (m) & 3 & 3 & 3 & 3 \\
Upper edge depth $(\mathrm{km})$ & 0.5 & 0.5 & 0.5 & 0.5 \\
\hline
\end{tabular}
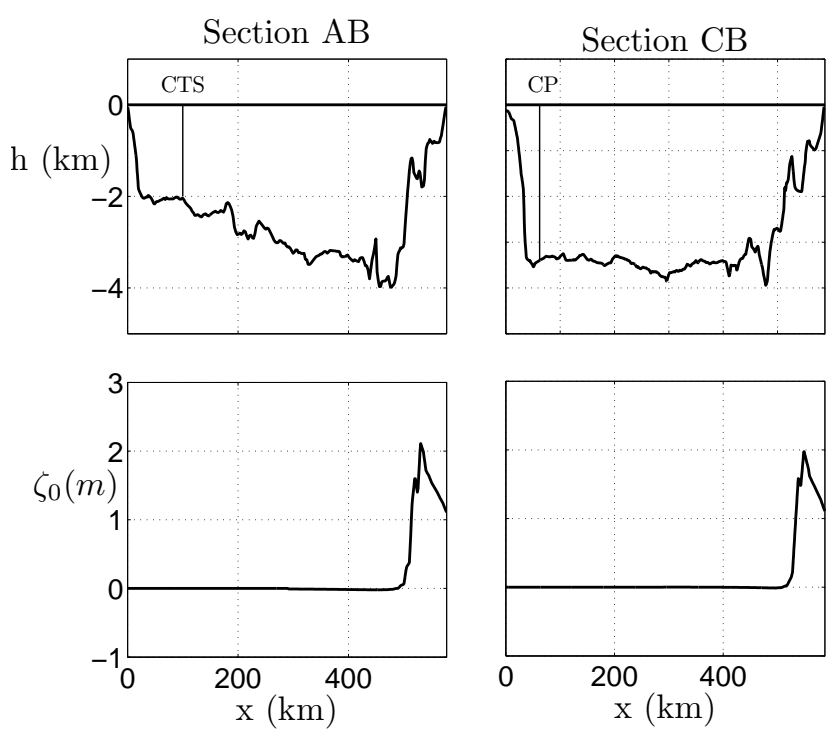

Figure 3. Upper panels: vertical sections AB and CB of Fig. 2. Section $\mathrm{AB}$ contains the location of the Catania submarine observatory, while section CB the Capo Passero Observatory. Lower panels: residual vertical sea bed displacement $\zeta_{0}$ relative to the above vertical section.

graphical simulations have been carried out in the domain of Fig. 1, which has been discretized by 2250000 triangular elements, using a maximum element mesh size of $1200 \mathrm{~m}$. In order to reproduce the hydro-acoustic wave propagation in the whole domain, the simulation covers a time of $720 \mathrm{~s}$, with a time step of $0.1 \mathrm{~s}$. The computational time was about $100 \mathrm{~h}$ on a high-speed computer equipped with 12 cores i7 $3.20 \mathrm{GHz}$ CPU and 64 GB RAM.

The results of the numerical model application in terms of free-surface elevation are represented in Fig. 5. At the time $t=0$ the earthquake occurs; then the hydro-acoustic wave travels in the Mediterranean Sea at the sound celerity in water, assumed constant as $1500 \mathrm{~m} \mathrm{~s}^{-1}$. Each $90 \mathrm{~s}$ a snapshot of the free-surface elevations is represented, showing that after $180 \mathrm{~s}$ the coasts of Libya (Africa) are reached by the hydro-acoustic waves, while after $360 \mathrm{~s}$ the acoustic signal arrives at the coasts of Tunisia and Italy. After about $500 \mathrm{~s}$
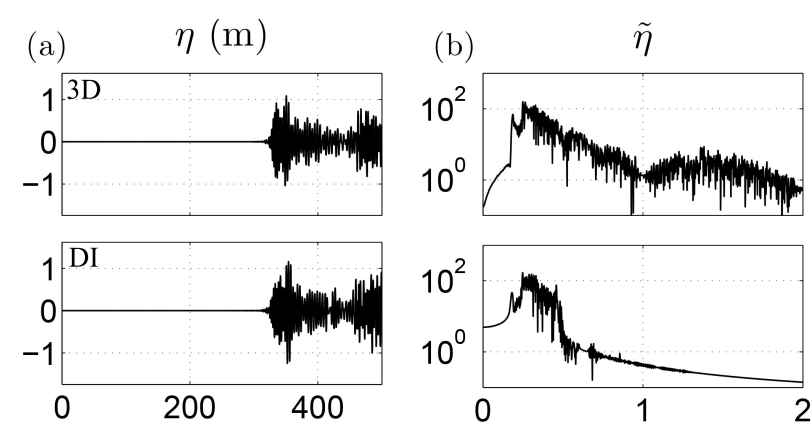

(c)

$\eta(\mathrm{m})$
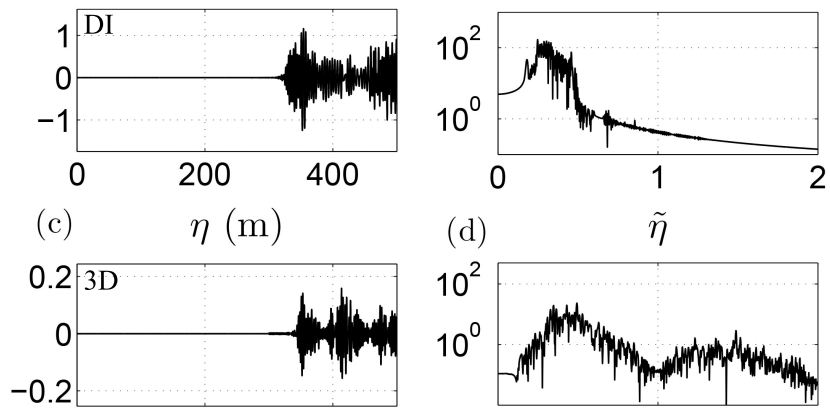

(d)
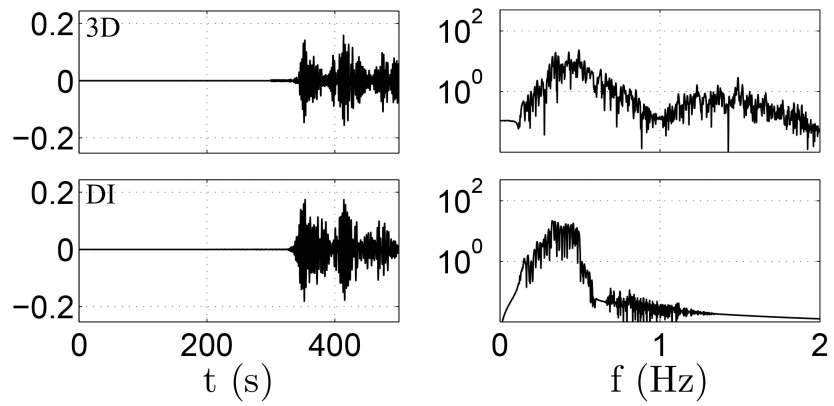

Figure 4. Free-surface time series (left column) and their relative frequency spectra (right column) at the CTS point (a and b) and CP point (c and d). Comparison between the solution of the 3-D reference model (upper plots) and the solution of the depth-integrated equation (lower plots).

the hydro-acoustic perturbation has covered almost all the domain. From the snapshots for $t>450 \mathrm{~s}$ it can be noted that the maximum values of free-surface oscillation are located around the areas of deeper waters, which occur, as can be seen in Fig. 1, south of Greece and between Sicily and Libya, in the middle of the numerical domain. The simulation results shown in Fig. 5 confirm that the hydro-acoustic signal does not propagate upslope (Nosov and Kolesov, 2007; Kadri and Stiassnie, 2012): the shallower water areas south-west of Sicily are not reached by the hydro-acoustic perturbation even after longer time from the earthquake occurrence.

At the two points where the submarine stations are located, CTS and CP, the simulated pressure perturbations associated 

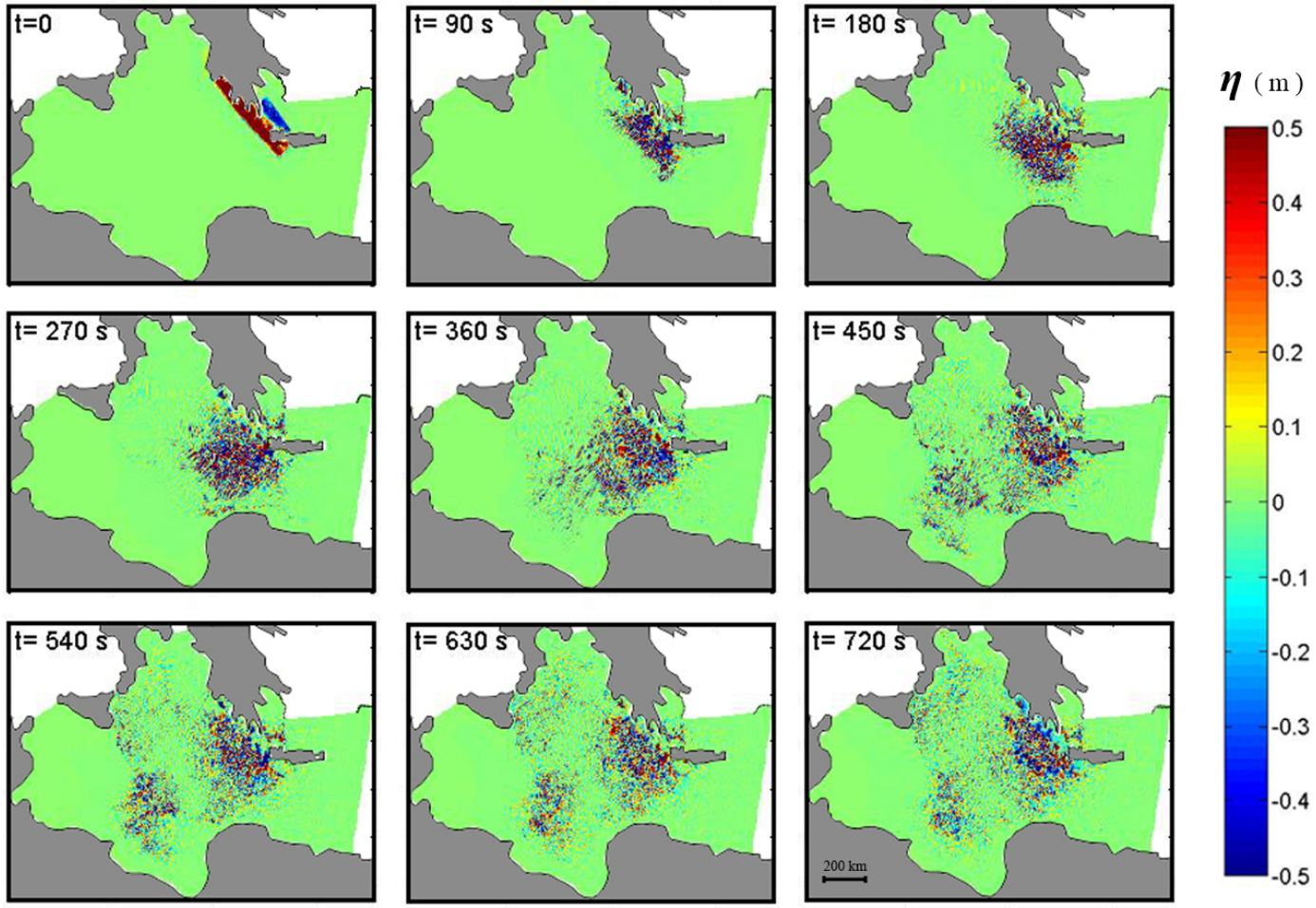

Figure 5. Snapshots of the free surface $(\eta)$ hydro-acoustic perturbation given by the AD 365 Crete earthquake. $t=0$ refers to the time of occurrence of the earthquake.
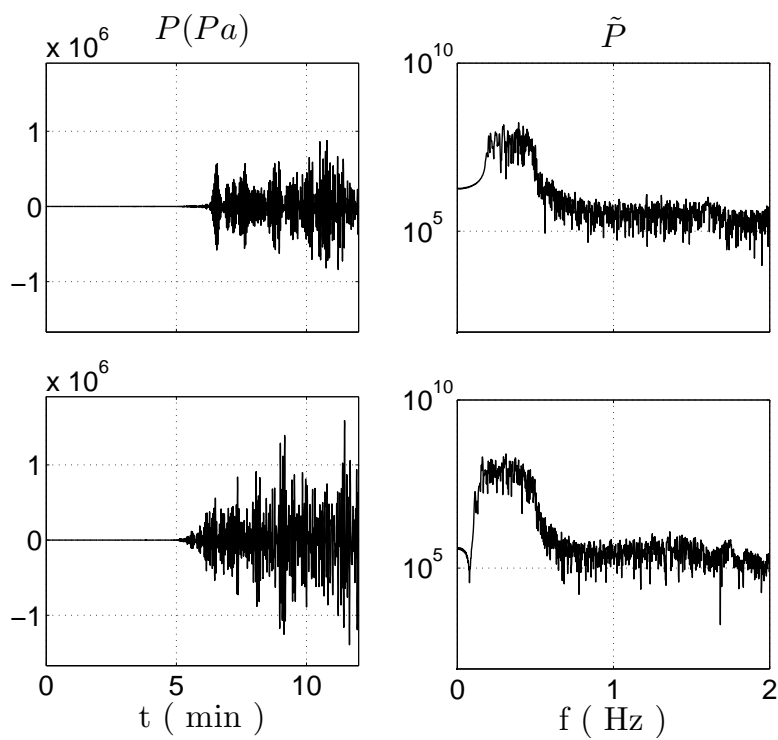

Figure 6. Pressure time series (left column) and their frequency spectra (right column) at Catania Test Site point (upper plots) and at Capo Passero point (lower plots), resulting from the numerical solution of the depth-integrated Eq. (5) over the whole domain.

with hydro-acoustic first mode are calculated at the sea bottom. Figure 6 represents the pressure time series (left column) and the frequency spectra (right column) at Catania
Test Site submarine station (upper plots) and at Capo Passero submarine station (lower plots). At both points the hydroacoustic signal arrives around $5 \mathrm{~min}$ after the earthquake occurrence, while the long free-surface tsunami waves arrive after about $40 \mathrm{~min}$, assuming a constant velocity of $\sqrt{g h}$ in a mean water depth of $2.5 \mathrm{~km}$.

Since most of the seabed motion occurs at a water depth of $3 \mathrm{~km}$, the generated hydro-acoustic waves oscillate at a frequency close to the cutoff value of the first hydro-acoustic mode, i.e., $f^{(1)}=c_{\mathrm{S}} / 4 \mathrm{~h}=0.12 \mathrm{~Hz}$. This signal is however filtered when traveling on shallower water depth, which allow the propagation only of higher-frequency waves (Kadri and Stiassnie, 2012). The model results, shown in term of frequency spectra in Fig. 6, confirm this water depth filtering effect: the CTS is located at a water depth of $2 \mathrm{~km}$, and therefore the cutoff frequency for propagating wave is $f^{(1)}=0.19 \mathrm{~Hz}$; the CP site is located at $3 \mathrm{~km}$ of water depth and records wave oscillation of $0.12 \mathrm{~Hz}$.

\subsection{The 1693 scenario}

On 11 January 1693 a powerful earthquake occurred offshore of the east coast of Sicily, Italy. This earthquake was preceded by a damaging foreshock on 9 January. It had an estimated magnitude of 7.2, one of the most powerful in Italian history, destroying at least 70 towns and cities and causing the death of about 60000 people. The earthquake was fol- 


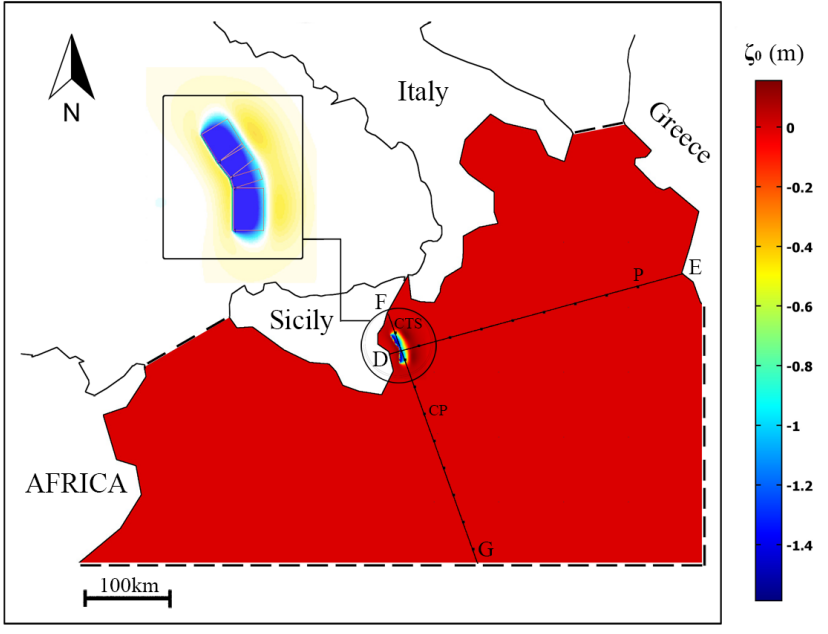

Figure 7. Residual vertical seabed dislocation, $\zeta_{0}(\mathrm{~m})$, for the 1693 Sicily earthquake, as reconstructed by Tonini et al. (2011)

lowed by tsunamis that devastated the coastal villages at the Ionian Sea coasts and in the Strait of Messina (Tinti et al., 2004). The strongest effects were concentrated around $\mathrm{Au}$ gusta, where the initial withdrawal of the water left the harbor dry, followed by a wave of at least $2.4 \mathrm{~m}$ height, possibly as much as $8 \mathrm{~m}$, that inundated part of the town. The maximum inundation of about $1.5 \mathrm{~km}$ was recorded at Mascali. The identification of the tsunami source is still an open issue; Tonini et al. (2011) carried out the Worst-case Credible Tsunami Scenario Analysis on the basis of a possible earthquake and a possible submerged landslide. For the analysis of the hydro-acoustic wave generation, the reconstructed earthquake scenario assumed by Tonini et al. (2011) has been chosen. The seismic parameters of Table 3 are taken as wave generation condition. Figure 7 shows the plan distribution of the vertical residual seabed motion in the overall numerical domain, which occurs downward, differently from the $\mathrm{AD}$ 365 Crete scenario.

Again, the depth-integrated model is tested by comparison with the 3-D solution at two vertical sections of the sea. The position of these sections is represented in Fig. 7, while Fig. 8 shows the water depth and the vertical residual seabed dislocation over the two sections. From the comparison between the solution of the complete 3-D equation and of the MSEWC, a good agreement is found over all the points of both sections. The results at Capo Passero site on the FG section and at a point close to the Greek coast (point P) on the DE section are presented.

Figure 9 shows free-surface elevation time series and frequency spectra at $\mathrm{CP}$ point (panels a and $\mathrm{b}$ ) and at $\mathrm{P}$ point, close to the Greek coast (panels c and d). In each panel the upper plot shows the 3-D reference model results, while the lower panel shows the solution of the MSEWC. The depthintegrated results in the time domain almost perfectly reproduce the solution of the 3-D mathematical problem. As
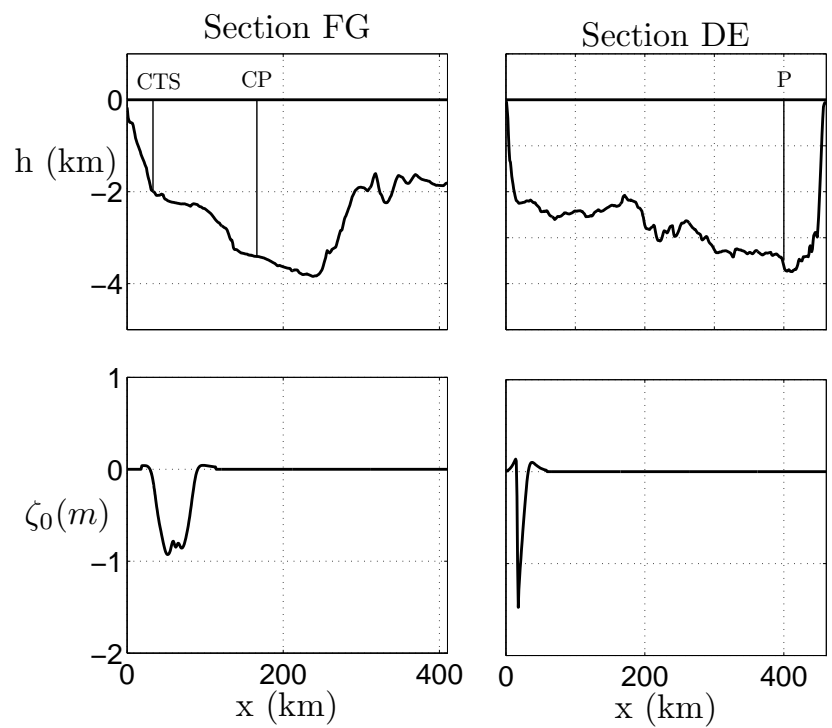

Figure 8. Upper panels: vertical sections FG and DE of Fig. 7. Lower panels: residual vertical seabed displacement $\zeta_{0}$ relative to the above vertical section.

it can be noted by looking at the spectra, the comparison is in a good agreement for the MSEWC reproduced frequency range $(0.15-0.3 \mathrm{~Hz})$. In the depth-integrated modeling the reproduction of frequencies out of this range, and relative to the second and higher hydro-acoustic mode, increases the computational time without providing a relevant improvement in the wave simulation. From the complete 3D simulation it is possible to notice clearly that the hydroacoustic signal during its propagation vibrates at the normal frequencies of the water layer where it has been generated, around $2 \mathrm{~km}$. The Capo Passero observation site is at a water depth of about $3 \mathrm{~km}$, deeper than the earthquake location and close to it; therefore the frequency spectrum at CP of Fig. 9 shows five peaks, which are relative to the first five hydro-acoustic modes that occur at the normal frequencies $f^{(n)}=0.19,0.56,0.9,1.31$ and $1.69 \mathrm{~Hz}$, accordingly to Eq. (4). Traveling far from the generation area the pressure perturbation loses this peaked frequency spectrum around the cutoff values, as can be noted at point $P$, around $350 \mathrm{~km}$ far from the earthquake.

The large-scale depth-integrated simulation has been carried out in the domain represented in Fig. 7, which has been discretized by means of triangular elements with a maximum size of $1200 \mathrm{~m}$, for a total of 1110000 elements. The time interval numerically reproduced is of $600 \mathrm{~s}$ after the earthquake occurrence and a time step of $0.1 \mathrm{~s}$ has been used. The computational time to reproduce this simulation was about $50 \mathrm{~h}$ using the same computer described before. 

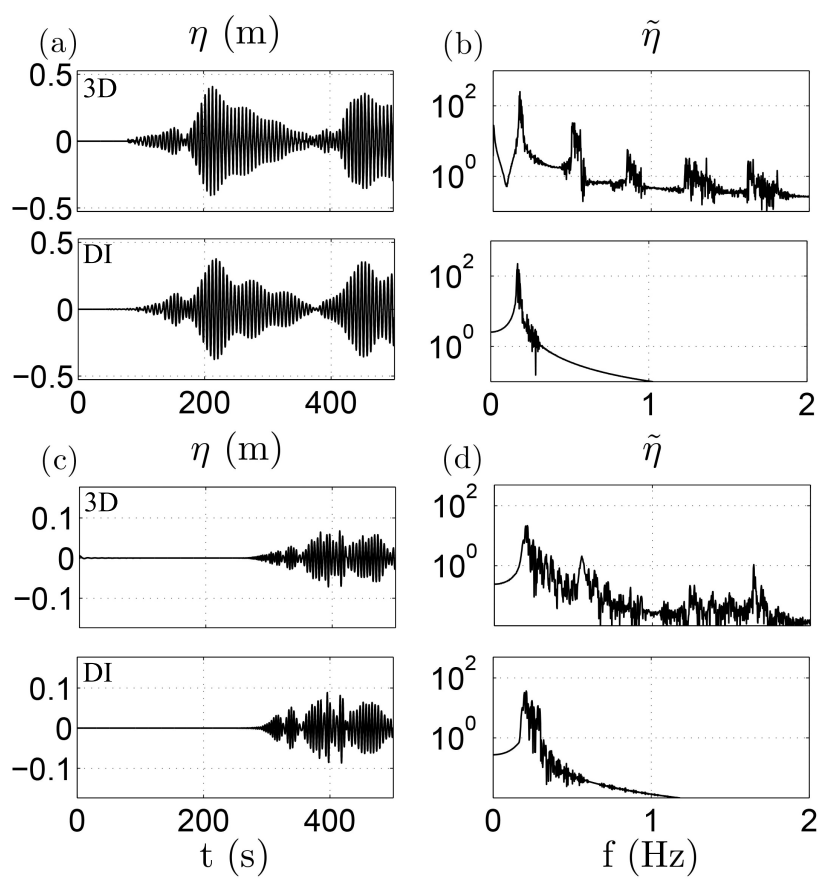

Figure 9. Free-surface time series (left column) and their relative frequency spectra (right column) at the $\mathrm{CP}$ point (a and b) and at $P$ point (c and d). Comparison between the solution of the 3-D reference model (upper plots) and the solution of the depth-integrated equation (lower plots).

Snapshots of the free-surface elevation from $t=0$ (earthquake occurrence) every $1 \mathrm{~min}$, up to $8 \mathrm{~min}$, are shown in Fig. 10. At time $t=4 \mathrm{~min}$ the hydro-acoustic wave reached the Greek coasts. After that time, the maximum values of the free-surface hydro-acoustic waves, around $6 \mathrm{~cm}$, are at the deeper waters, while the propagation in the shallower areas does not occur.

The results of the large-scale depth-integrated simulation are presented at the two observation points, CTS and CP, in terms of bottom pressure. Figure 11 shows the pressure signals in the time and the frequency domain, at the two points CTS (upper plots) and CP (lower plots). The CTS point lies above the earthquake, while CP point is about $100 \mathrm{~km}$ from the epicenter; hence the hydro-acoustic signal arrives after about $1 \mathrm{~min}$ from the earthquake occurrence. Despite the time of arrival and a greater amplitude of the waves at CTS just after the earthquake occurrence, the two pressure signals are very similar. The generated signal has a peak frequency around $0.19 \mathrm{~Hz}$, since the waves are generated at a water depth of $2 \mathrm{~km}$, and at the same frequency associated propagating modes are at the deeper $3 \mathrm{~km}$ water depth $(\mathrm{CP})$.

\section{Discussion and conclusions}

The Mediterranean seabed is affected by intense seismic activities. The potential tsunamis can therefore be destructive since the coasts are densely populated and very close: the travel time of tsunami wave towards the coast is in the order of few minutes up to $1 \mathrm{~h}$. Rapid detection of the tsunami generation in this region is mandatory for future development of early warning systems, and the use of the hydro-acoustic signals can cope with this necessity. This appealing feature of hydro-acoustic waves has recently been considered by Renzi and Dias (2014) for the wave field generated by a localized surface pressure disturbance; their work could lead to the design of innovative alert systems for coastal flooding management, based on the measurements of hydro-acoustic wave.

The proposed model reproduces the mechanism of propagation of hydro-acoustic waves due to a sudden bottom displacement associated with earthquakes, solving the mildslope equation in weakly compressible fluid of Sammarco et al. (2013). In order to develop innovative warning procedures based on these acoustic signals, the correct modeling of wave field, in weakly compressible fluid, at realscale domains is extremely important. The use of a depthintegrated equation allows the simulation in large geographical areas, such as the Mediterranean Sea, where the 3-D modeling requires huge computational times. The depthintegrated equation has been already validated by Sammarco et al. (2013); however, in this paper an application and validation in real bathymetry has been developed. The full modeling of the hydro-acoustic waves field generated by two historical tsunamigenic earthquake scenarios in the Mediterranean Sea has been carried out.

The numerical model results highlight that the seabed motion energy is transferred to hydro-acoustic waves mainly at the frequencies just after the cutoff values of each acoustic mode. Close to the generation area the frequency spectra clearly show energy peaks for each acoustic mode, while during wave propagation the hydro-acoustic energy will distribute along frequencies higher than the cutoff values. The simulation of the 1693 earthquake scenario shows that $100 \mathrm{~km}$ far from the epicenter, at Capo Passero observation site, the hydro-acoustic waves recorded still allow the identification of the energy associated with each acoustic mode. The numerical simulations confirm that the first mode is the one which carries most of the energy. The hydro-acoustic modes propagate undisturbed in water layers equal to or deeper than the one where they have been generated. As the waves propagate in shallower water depth, characterized by higher cutoff frequencies, the components with frequencies lower than the cutoff become evanescent: when hydroacoustic waves propagate towards shallower sea depth, the water layer acts as a frequency filter. 

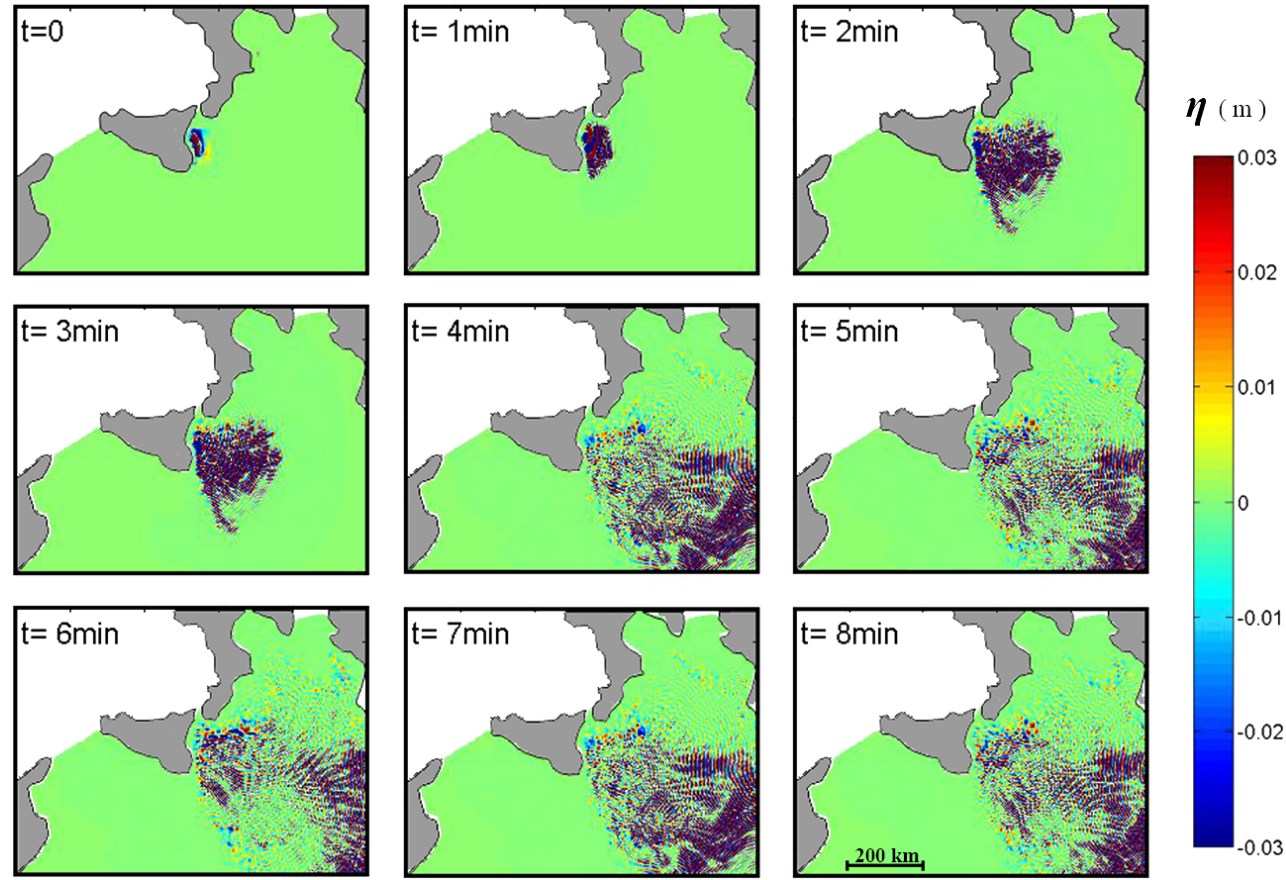

Figure 10. Snapshots of the free surface $(\eta)$ hydro-acoustic perturbation given by the 1693 Catania (Sicily) earthquake. $t=0$ refers to the time of occurrence of the earthquake.
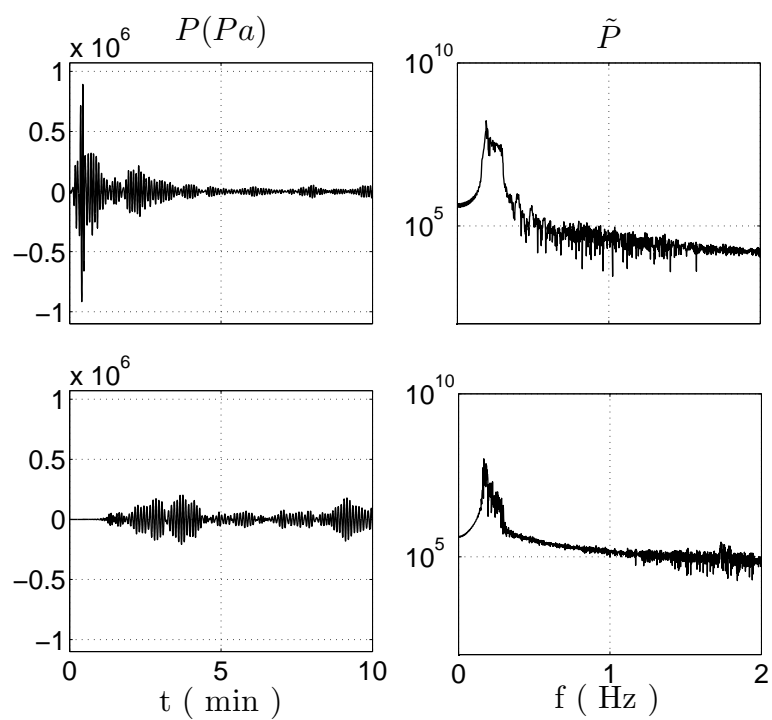

Figure 11. Pressure time series (left column) and their frequency spectra (right column) at Catania point (upper plots) and at Capo Passero point (lower plots), resulting from the numerical solution of the depth-integrated Eq (5) over the whole domain.

It is worth mentioning that landslides are the second major cause of tsunami generation. The present model is in principle able to reproduce a submarine motion of rigid landslide. However, due to the occurrence of landslides mainly near the shore, and since during its motion the landslide would gener- ated pressure waves at different water depths, further studies need to be addressed on this topic.

To implement innovative tsunami early warning systems based on measurement and analysis of hydro-acoustic signals, the complete modeling of hydro-acoustic waves in real bathymetry has proven to be extremely useful. The numerical model simulations show that the hydrophones must be placed in waters deep enough to record larger frequency ranges and, if possible, not shielded by seamounts. However these instruments should be connected to the shore by submarine cables to guarantee fast data transmission; therefore their location can not be too far from the coastline. Hence the model can help choose the right positioning of the hydrophone system. For the portion of the Mediterranean Sea here analyzed, the numerical simulation results suggest that offshore of the $\mathrm{Si}$ cilian east coast, where the instruments have been placed, and offshore of the Greek west coast, the waters are deep enough to record and identify the hydro-acoustic waves generated by seismic activities in both the Hellenic arc and the Ionian Sea (southern Italy).

Acknowledgements. This work was carried out under the research project FIRB 2008-FUTURO IN RICERCA ("Design, construction and operation of the Submarine Multidisciplinary Observatory experiment"), funded by the Italian Ministry for University and Scientific Research (MIUR). We wish to thank G. Riccobene, scientific coordinator of the mentioned FIRB project, for the useful discussions. 
Edited by: M. Gonzalez Rodríguez

Reviewed by: E. Renzi and one anonymous referee

\section{References}

Abdolali, A., Cecioni, C., Bellotti, G., and Sammarco, P.: A depth-integrated equation for large scale modeling of tsunami in weakly compressible fluid, Coast. Engin. Proc., 1, doi:10.9753/icce.v34.currents.9, 2014.

Abdolali, A., Cecioni, C., Bellotti, G., and Kirby, J. T.: Hydroacoustic and tsunami waves generated by the 2012 Haida Gwaii earthquake: modeling and in-situ measurements, J. Geophys. Res. Oc., 120, 958-971, doi:10.1002/2014JC010385, 2015a.

Abdolali, A., Kirby, J. T., and Bellotti, G.: Depth integrated equation for hydro-acoustic waves with bottom damping, J. Fluid Mechan., 766, doi:10.1017/jfm.2015.37, 2015b.

Ardhuin, F. and Herbers, T.: Noise generation in the solid Earth, oceans and atmosphere, from nonlinear interacting surface gravity waves in finite depth, J. Fluid Mechan., 716, 316-348, 2013.

Berkhoff, J. C. W.: Computation of combined refraction-diffraction, ASCE, Vancouver, Canada, 1, 471-490, 1972.

Bolshakova, A., Inoue, S., Kolesov, S., Matsumoto, H., Nosov, M., and Ohmachi, T.: Hydroacoustic effects in the 2003 Tokachi-oki tsunami source, Russ. J. Earth Sci., 12, ES2005, doi:10.2205/2011ES000509, 2011.

Cecioni, C. and Bellotti, G.: Inclusion of landslide tsunamis generation into a depth integrated wave model, Nat. Hazards Earth Syst. Sci., 10, 2259-2268, doi:10.5194/nhess-10-2259-2010, 2010 a.

Cecioni, C. and Bellotti, G.: Modeling tsunamis generated by submerged landslides using depth integrated equations, Appl. Oc. Re., 32, 343-350, 2010b.

Cecioni, C., Bellotti, G., Romano, A., Abdolali, A., and Sammarco, P.: Tsunami early warning system based on real-time measurements of hydro-acoustic waves, Proc. Engin., 70, 311-320, 2014.

Chierici, F., Pignagnoli, L., and Embriaco, D.: Modeling of the hydroacoustic signal and tsunami wave generated by seafloor motion including a porous seabed, J. Geophys. Res. Oc. (19782012), 115, C03015, doi:10.1029/2009JC005522, 2010.

Chung, J. and Hulbert, G.: A time integration algorithm for structural dynamics with improved numerical dissipation: the generalized- $\alpha$ method, J. Appl. Mechan., 60, 371-375, 1993.
Eyov, E., Klar, A., Kadri, U., and Stiassnie, M.: Progressive waves in a compressible-ocean with an elastic bottom, Wave Motion, 50, 929-939, 2013.

Jansen, K. E., Whiting, C. H., and Hulbert, G. M.: A generalized- $\alpha$ method for integrating the filtered Navier-Stokes equations with a stabilized finite element method, Comput. Method. Appl. M., 190, 305-319, 2000.

Kadri, U. and Stiassnie, M.: Acoustic-gravity waves interacting with the shelf break, J. Geophys. Res. Oc. (1978-2012), 117, C03035, doi:10.1029/2011JC007674, 2012.

Miyoshi, H.: Generation of the tsunami in compressible water (Part I), J. Oceanogr. Soc. Jpn., 10, 1-9, 1954.

Nosov, M. A.: Tsunami generation in compressible ocean, Physics and Chemistry of the Earth, Part B: Hydrology, Oceans Atmos., 24, 437-441, 1999.

Nosov, M. A. and Kolesov, S. V.: Elastic oscillations of water column in the 2003 Tokachi-oki tsunami source: in-situ measurements and 3-D numerical modelling, Nat. Hazards Earth Syst. Sci., 7, 243-249, doi:10.5194/nhess-7-243-2007, 2007.

Okada, Y.: Surface deformation due to shear and tensile faults in a half-space, Bull. Seismol. Soc. Am., 75, 1135-1154, 1985.

Renzi, E. and Dias, F.: Hydro-acoustic precursors of gravity waves generated by surface pressure disturbances localised in space and time, J. Fluid Mechan., 754, 250-262, 2014.

Sammarco, P., Cecioni, C., Bellotti, G., and Abdolali, A.: Depth-integrated equation for large-scale modelling of lowfrequency hydroacoustic waves, J. Fluid Mechan., 722, R6, doi:10.1017/jfm.2013.153, 2013.

Sells, C. C. L.: The effect of a sudden change of shape of the bottom of a slightly compressible ocean, Philosophical Transactions of the Royal Society of London. Series A, Mathemat. Phys. Sci., 258, 495-528, 1965.

Shaw, B., Ambraseys, N., England, P., Floyd, M., Gorman, G., Higham, T., Jackson, J., Nocquet, J.-M., Pain, C., and Piggott, M.: Eastern Mediterranean tectonics and tsunami hazard inferred from the AD 365 earthquake, Nature Geosci., 1, 268-276, 2008.

Stiassnie, M.: Tsunamis and acoustic-gravity waves from underwater earthquakes, J. Engin. Mathemat., 67, 23-32, 2010.

Tinti, S., Maramai, A., and Graziani, L.: The new catalogue of Italian tsunamis, Nat. Hazards, 33, 439-465, 2004.

Tonini, R., Armigliato, A., Pagnoni, G., Zaniboni, F., and Tinti, S.: Tsunami hazard for the city of Catania, eastern Sicily, Italy, assessed by means of Worst-case Credible Tsunami Scenario Analysis (WCTSA), Nat. Hazards Earth Syst. Sci., 11, 1217-1232, doi:10.5194/nhess-11-1217-2011, 2011.

Yamamoto, T.: Gravity waves and acoustic waves generated by submarine earthquakes, Internat. J. Soil Dynam. Earth. Engin., 1, 75-82, 1982. 